International Journal of Computer Networks \& Communications (IJCNC) Vol.4, No.3, May 2012

\title{
On Throughrut Performance of DeCode AND FORWARD COOPERATIVE RELAYING WITH PACKET COMBINING AND ARQ
}

\author{
Rajendrakumar Patil ${ }^{1}$ \\ ${ }^{1}$ Department of Electrical Engineering, Indian Institute of Technology, Bombay, \\ India. \\ rapdee.iitb.ac.in
}

\begin{abstract}
Cooperative Diversity can be used by wireless nodes to take the benefit of broadcast nature and inherent spatial diversity of wireless channel to relay messages for each others. This helps to propagate redundant signals over multiple paths, which can be used to combat the channel noise and fading effects. We investigate the use of packet combining in cooperative diversity scenario. In our approach we exploit this inherent spatial diversity alongwith the inherent time diversity in ARQ retransmissions. Further different channels have different fade levels or channel conditions. Maximal Ratio Combining of packets at the receiver improves the total Signal to Noise Ratio at the receiver, improving the throughput in wireless channel. Decode and Forward Relay can allow the opportunistic relay of the packets. In opportunistic relay of packets, the packet is relayed only when the direct transmission from source to destination fails. This saves the resources by avoiding the relay of packets, when not required. In this paper we propose the relaying scheme with packet combining at the destination. We investigate the BER and throughput performance of the scheme in AWGN and fading channel with and without MRC combining and compare them.
\end{abstract}

\section{KEYWORDS}

Packet Combining, ARQ, DF Relay, Relay Networks, $M R C$

\section{INTRODUCTION}

Wireless networks are becoming very popular now a day due to advances in the new generation of wireless technology, which offer more flexibility and mobility. New generation wireless networks have improved data rate and connectivity. The wireless network is inherently broadcast in nature. Hence it is possible for wireless nodes to forward each other's messages cooperatively to improve throughput, coverage, capacity and reliability of the network. This cooperative relaying has changed the design concept of wireless network. The cooperative relay technique is deployed in next generation wireless standards like IEEE 802.16 (WiMax), LTE, Cognitive Radio and other applications. IEEE 802.16j supports relay technology to improve the throughput at the cell edge users, to temporary locations and to transport vehicles. To improve the cell-edge throughput and to extend the coverage of high data rates, LTE Advanced-standard specified by 3GPP (Third Generation Partnership project) uses cooperative relay technology. The cooperative relay technology was enhanced by the recent works of Laneman, Tse and Wornell. They have proposed the relaying techniques in [1]. In our approach we propose a incremental relaying scheme with one relay using Decode and Forward (DF) relaying. This scheme uses single bit feedback from destination indicating success or failure of transmission to forward the message by relay to destination in next phase. Here the transmission is performed 
International Journal of Computer Networks \& Communications (IJCNC) Vol.4, No.3, May 2012

in two phases. In first phase source transmits message to destination and if it fails, relay transmits the message to destination in second phase. Thus second phase do not occur if direct transmission is successful in first phase. This leads to efficient utilization of resources.

In [2], we considered ARQ on a point to point link. We used packet combining of erroneous packets and adaptation of packet size to channel condition to maximize the throughput. This work was not in the framework of cooperative ARQ, as all the copies of the packet are coming from the transmitter node and not from the relay node. The work here considers a cooperative ARQ where the retransmissions can be performed by cooperative Relay nodes. The transmission is overheard by all the relay nodes in the range of transmitter and they store the incoming packet in the buffer at relay. When the packet is successfully received by the receiver it sends an ACK and sends NACK when it is not successfully received. This ACK/NACK is received by the relay stations also. When a relay station gets ACK, it removes the packet stored in buffers. When it gets NACK, it retransmits the packet stored in buffers, instead of retransmissions by a transmitter. If all the cooperative relay nodes do not have correct copy of a packet, the receiver will perform packet combining to retrieve the correct copy. In conventional cooperative diversity networks, relays transmit the source signal to the destination irrespective of the channel condition. However, in our proposed method the relay transmits the source signal to the destination only when the source to destination transmission fails. This failure in transmission is sensed by the relay by hearing the negative Acknowledgement (NACK). The relay is used opportunistically (using the relay only when the end to end throughput using relay is greater than the throughput of direct link) based on the limited feedback from the receiver. When multiple relays are used, radio resources are required to maintain the orthogonality between them. The relay cannot transmit and receive simultaneously. Some time division multiplexing is required for receiving and transmitting at relay. Due to limited availability of radio resources, we consider only one relay model. The paper is organized as follows. Section II discusses the related work. Section III defines the system model and describes the proposed algorithm in the cooperative relay scenario. Section IV discusses about the rate of the proposed scheme. The simulation results are discussed in Section V and the conclusions are given in Section VI.

\section{LITERATURE REVIEW}

Significant work on cooperative relaying has been done recently. There is plenty of literature available on analytical performance comparison of Amplify and Forward (AF), Decode and Forward (DF) and other cooperative protocols. In [1] the strategies for cooperative relaying are outlined and the performance characterization is done. The fixed AF and DF relaying, Selection Relaying and Incremental Relaying protocols are analyzed and compared in terms of their outage behavior. The end-to-end performance of incremental relaying cooperative diversity networks using amplify and forward (AF) relays is studied in [3]. They concluded that the cooperation significantly improves the BER performance in comparison with the direct transmission and incremental relaying improves the channel utilization. The modeling and performance analysis of an amplify-and-forward cooperative diversity wireless network is done in [4]. They developed a Markov-based model which, quantifies equivalent SNR saving per additional cooperative node to maintain the packet delivery delay, computes the minimum transmission power and the packet delivery probability.

In all these work mentioned above the cooperation is at physical layer. They do not consider cross layer approach in which the packet length at MAC layer is adapted based on the channel condition. They also do not exploit the inherent time diversity in ARQ retransmissions to recover a packet by MRC combining of the packets with their retransmissions. Also they do not consider the rate adaptation based on channel condition in cooperative relay networks. In our work we use cross layer approach to exploit the inherent time diversity in ARQ retransmissions 
International Journal of Computer Networks \& Communications (IJCNC) Vol.4, No.3, May 2012

to recover a packet by MRC combining of the packets with their retransmissions. We employ cross layer packet length adaptation and rate adaptation using AMC at the transmitter node.

The throughput performance of fixed relaying cooperative wireless networks using adaptive modulation over Rayleigh fading channel is studied in [5]. They showed that the throughput of the wireless network can be enhanced further if adaptive modulation is used along with cooperative diversity. In [6], the authors presents a cross layer link adaptation design for cooperative ARQ based relay channel in which both source and relay employ AMC and power adaptation at the physical layer. But they do not consider packet combining in their work and use fixed relaying. In [7], the authors use adaptive modulation for Demodulate and Forward (DMF) cooperative fixed relay system to maximize network throughput under the constraint of the Bit Error Rate (BER) requirement. In packet combining the receiver can combine multiple erroneous transmissions to recover a correct packet. In [8] cooperative packet recovery is done using the physical layer information like a confidence measure on their 0-1 decision for each bit. The confidence values across multiple faulty receptions are combined to recover a clean packet. The rate and power adaptation technique is used in [9] to maximize the throughput in fixed DF cooperative relay networks when limited feedback links exists to the transmitter node. In this paper, the authors found that significant performance improvement is possible using cooperative techniques over traditional point to point communication.

The authors propose a relay ARQ and destination ARQ strategies in [10] for relay cooperative system using fixed Decode and Forward (DF) relay. The BER performance of the strategies is analyzed. The performance gain in their strategy over non relay system is not significant when channel condition is good. They did not consider the opportunistic or incremental relay strategies, which saves the system resources when the channel condition is good.

\section{SYSTEM MODEL}

We apply the packet combining ARQ scheme [2] to relay networking. The work here considers a packet combining ARQ scheme, where the retransmissions can be performed by cooperative Relay nodes. The transmission is overheard by all the relay nodes in the range of transmitter and they store the incoming packet in the buffer at relay. When a packet is successfully received by the receiver, it sends an ACK and sends NACK when it is not successfully received. This ACK/NACK is received by the relay stations also. When a relay station gets ACK, it removes the packet stored in buffers. When it gets NACK, it retransmits the packet stored in buffers, instead of retransmissions by a transmitter. If all the cooperative relay nodes do not have correct copy of a packet, the receiver will perform packet combining to retrieve the correct copy. In conventional cooperative diversity networks, relays transmit the source signal to the destination irrespective of the channel condition. However, in our proposed method, the relay transmits the source signal to the destination only when the source to destination transmission fails. This is sensed by the relay by hearing the negative Acknowledgement (NACK). The relay is used opportunistically (using the relay only when the end to end throughput using relay is greater than the throughput of direct link) based on the limited feedback from the receiver.

When multiple relays are used, radio resources are required to maintain the orthogonality between them. The relay cannot transmit and receive simultaneously. Some time division multiplexing is required for receiving and transmitting at relay. Due to limited availability of radio resourses, we consider only one relay model.

We consider a three node network uplink scenario as shown in the Figure 1. The three nodes are MS (Mobile Station), RS (Relay Station) and BS (Base Station). 


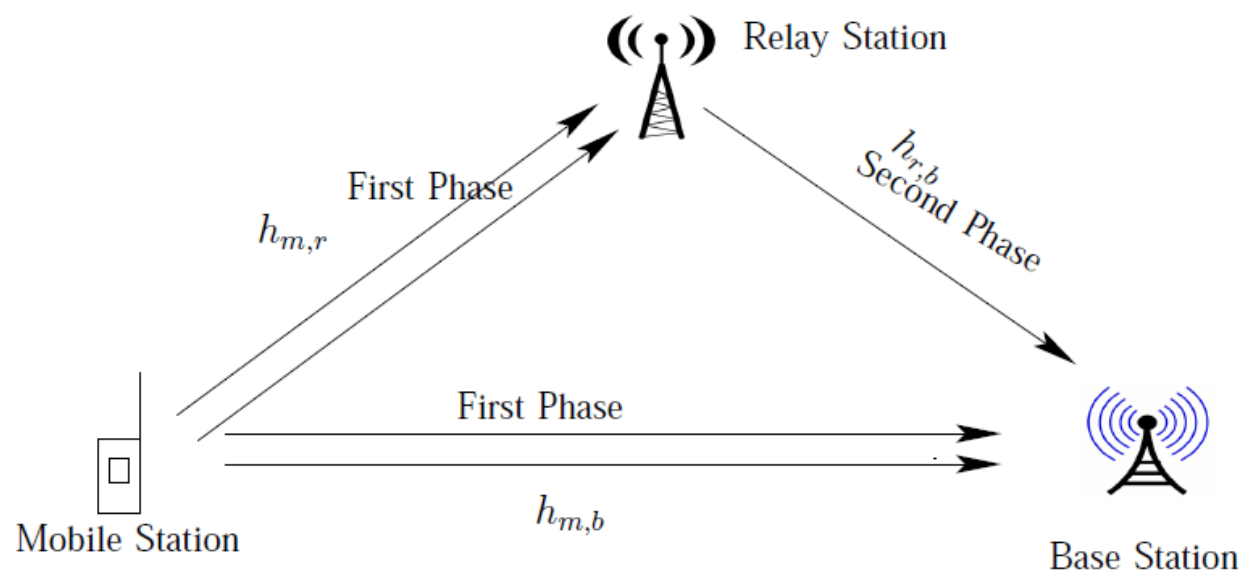

Figure 1. Cooperative Relay Scenario

In this model, we assume i.i.d Rayleigh fading channel between MS-RS, MS-BS and RS-BS. The relay used in the model is assumed to be Decode and Forward (DF) incremental relay. In DF relaying, the relay decodes the received signal and forwards it to the destination [11]. In this process, DF relay re-encode the signal and then transmits to the destination. Incremental relay transmits the received information to the destination only when the direct transmission from source to destination fails. Incremental relaying protocol exploits the limited feedback from the destination, which is a single bit indicating the success or failure of the direct transmission. In cooperative relay network, the transmission takes place in two phases. Here, MS transmits a packet in first phase, which is received by the RS and BS. When the direct transmission to BS is successful, BS sends ACK which is received by MS and RS. On receiving ACK from BS, RS do not forward the received packet and MS transmits the next packet. When the direct transmission is in error, BS stores the erroneously received packet and sends NACK which is received by the MS and RS. On receiving NACK, RS forwards the packet to BS in second phase. BS performs MRC combining on the received copies of the packet. If the MRC combined packet is in error, BS requests the retransmission of the packet by sending NACK and then MS retransmits the packet. Here, occurrence of the second phase depends on the successful or unsuccessful reception of the packet in the first phase. If the transmission is successful in first phase, the second phase do not occur. In this type of protocol, the information is repeated rarely. In other relaying schemes, the same information is transmitted twice over the entire cooperative transmission period, which leads to inefficient use of bandwidth. Hence there is a saving of bandwidth in incremental relaying scheme. In this model, we assume error free ACK/NACK.

We assume the transmitted signal from mobile station to be $x$ with unit energy. Here, we assume transmission by MS and RS at fixed power $P$. In this model, $y_{m, b}$ is the Signal received at the base station from mobile station (end-to-end-signal), $y_{m, r}$ is the Signal received at the relay station from mobile station, $n_{m, r}$ is the Additive noise in the MS-RS link with variance $=\sigma_{\mathrm{r}}^{2}, n_{m, b}$ is the Additive noise in the MS-BS link with variance $=\sigma_{\mathrm{b}}{ }^{2}, h_{m, r}$ is the channel coefficient on the MS-RS link, $h_{m b}$ is the channel coefficient on the MS-BS link, $h_{r, b}$ is the channel coefficient on the RS-BS link. The additive noise is zero mean complex Gaussian random variable with variance $=\mathrm{N}_{0}=\sigma_{\mathrm{r}}{ }^{2}=\sigma_{\mathrm{b}}{ }^{2}$. Then $y_{m, b}$ and $y_{m, r}$, the received signals at BS and RS respectively, are given by, 
International Journal of Computer Networks \& Communications (IJCNC) Vol.4, No.3, May 2012

$$
\begin{aligned}
& y_{m, b}=\sqrt{P} h_{m, b} x+n_{m, b} \\
& y_{m, r}=\sqrt{P} h_{m, r} x+n_{m, r} .
\end{aligned}
$$

SNR at the Transmitter side is given by $\gamma=\frac{P}{N_{0}}$. SNR on the MS to BS link is $\gamma_{m, b}=\frac{P}{N_{0}}\left|h_{m, b}\right|^{2}$. Signal Received at the Destination (BS) from relay is given by $y_{r, b}$. We assume that the noise terms $n_{m, r}$ and $n_{r, b}$ are independent. We also assume that the channel coefficients $h_{m, r}, h_{r, b}$ and $h_{m, b}$ are available at all the nodes.

The destination (base station) combines the received signal from the mobile station (sent in first phase) and the signal from relay station (sent in second phase). It MRC combines the signals to detect the transmitted symbol. MRC is the optimal combining technique which maximizes the overall SNR at the output of the combiner. The MRC combiner requires the coherent detector that have knowledge of all channel coefficients. The SNR at the output of the MRC is the sum of the received SNRs from both the branches. The output of MRC at the BS is given by,

$$
y=a_{1} y_{m, b}+a_{2} y_{r, b}
$$

where weights $a_{1}$ and $a_{2}$ are determined to maximize the SNR at the output of combiner. We assume that fading coefficients are circularly symmetric, zero mean, complex Gaussian random variables which model the Rayleigh fading [12]. The MS-BS, MS-RS and RS-BS links undergo independent Rayleigh fading.

\section{RATE OF THE PROPOSED SCHEME}

In the Selective DF relaying scheme applied here, if the SNR at the relay increases above certain threshold, the relay decodes the received signal correctly and forwards the decoded information to the destination in phase II. If the SNR at the relay falls below the threshold, the relay is idle. If the source relay SNR is below threshold, the relay can not decode the message correctly and hence in Selective DF relaying scheme, the source is allowed to transmit in phase II if the relay is not able to decode the message correctly. To get an end to end rate of R, the message must be encoded with a rate of $2 \mathrm{R}$ in cooperative transmission scenario. Hence in phase-I, the MS encodes the message with a rate $2 \mathrm{R}$. Decoding at the relay is correct if the transmission rate is less than or equal to the capacity of the MS-RS link i.e. $2 R \leq \log _{2}\left(1+\gamma_{m, r}\right)$.

The capacity of the MS-RS link is given by,

$$
C_{M S-R S}\left(\gamma_{m, r}\right)=\log _{2}\left(1+\gamma_{m, r}\right) \text { bits/channel use. }
$$

In this equation, $\gamma_{m, r}=\frac{P\left|h_{m, r}\right|^{2}}{\sigma_{r}{ }^{2}}$ 
International Journal of Computer Networks \& Communications (IJCNC) Vol.4, No.3, May 2012

From the above description of incremental relaying, it is clear that the rate is random in this protocol. If the first phase is successful, the rate is $2 \mathrm{R}$, if the first phase transmission is in outage, the rate is $\mathrm{R}$. The average rate of transmission is given by,

$$
\bar{R}=2 R \operatorname{Prob}\left(\gamma_{s, d} \geq 2^{2 R}-1\right)+R \operatorname{Prob}\left(\gamma_{s, d} \leq 2^{2 R}-1\right)
$$

Suppose the rate of transmission at MS is R, the outage occurs when $R>C_{M S-R S \text {. Incremental }}$ relaying scheme has higher bandwidth efficiency because the phase II of cooperative transmission occurs only when the transmission fails in phase-I. In this scheme, when the transmitted signal is $\mathrm{x}$, the received signal at BS and RS is given by equation (1). Here, $x$ may be a block of symbols. The transmission in phase-I is successful when $2 R<\log _{2}\left(1+\gamma_{m, b}\right)$, where $\gamma_{m, b}$ is the SNR of the direct link from MS to BS and is given by $\gamma_{m, b}=\frac{P\left|h_{m, b}\right|^{2}}{\sigma_{b}{ }^{2}}$. In this case end to end rate is $2 \mathrm{R}$. When the direct transmission is in outage, i.e. $2 R>\log _{2}\left(1+\gamma_{m, b}\right)$, the destination will send a NACK to inform the MS and RS about the unsuccessful transmission. In this case RS will forward the signal to BS in phase-II. The received signal at BS is given by, $y_{r, b}=h_{r, b} \sqrt{P} x+n_{r, b}$.

The signal received in phase II is,

$$
\begin{aligned}
& y_{r, b}=h_{r, b} \sqrt{P} x+n_{r, b}, \quad \text { if } \gamma_{m, r} \geq 2^{2 R}-1 \\
& y_{m, b}=h_{m, b} \sqrt{P} x+n_{m, b} \text { if } \gamma_{m, r} \leq 2^{2 R}-1
\end{aligned}
$$

The signals received in phase-I and phase-II are MRC combined at the BS . We assume that the source or relay address is appended to the packet in phase II which will enable the destination to identify the transmitter and determine the weighing factor for MRC. In this case the end to end rate is R. The SNR at the output of the MRC combiner is given by,

$$
\begin{aligned}
\gamma & =\gamma_{m, b}+\gamma_{r, b} \\
\text { where } \gamma_{r, b} & =\frac{P\left|h_{r, b}\right|^{2}}{\sigma_{d}^{2}}
\end{aligned}
$$

\section{SIMULATION RESULTS AND DISCUSSION}

We assume fix rate transmission at MS and RS. The packet size is also fixed. Simulation results of the incremental relay scheme with packet combining is first compared with the fixed relaying scheme with and without packet combining. The Pseudo code for the proposed relay scheme with packet combining is given in Algorithm 1. 


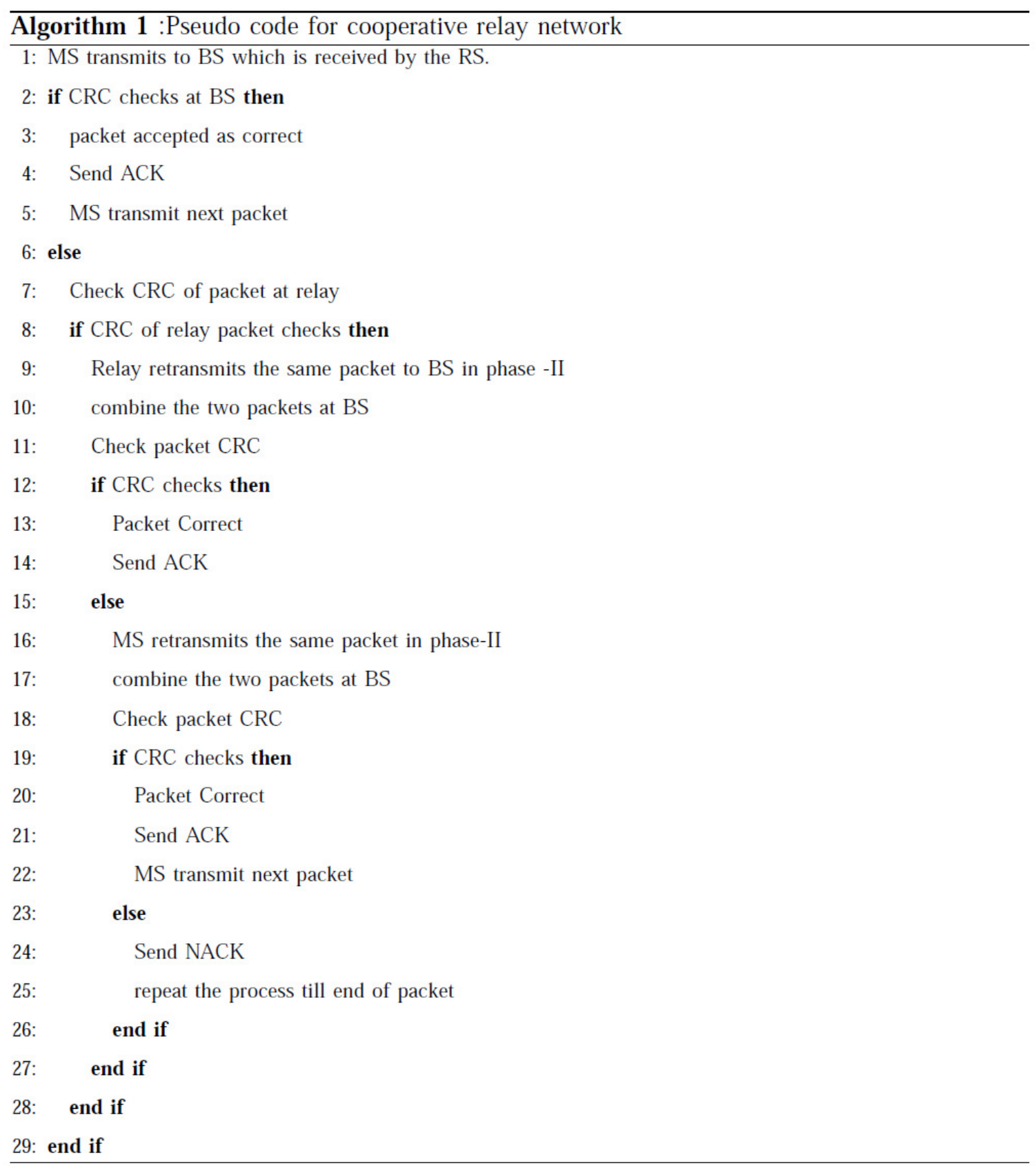

First we simulate a three node DF relay with fixed relaying and investigate the BER results. Here, our aim is to study the BER performance with relay and packet combining, then we investigate the throughput performance with our proposed scheme

\subsection{BER of three Node DF Relay with Fixed Relaying}

First we simulate the three node cooperative relay network using the Decode and Forward (DF) relay. In DF relaying there is processing capability introduced in the relay for decoding the incoming signal and then retransmit the signal to the receiver. Here, fixed relaying is used. In fixed relaying, the relay node transmits the received signal to base station in all channel 
International Journal of Computer Networks \& Communications (IJCNC) Vol.4, No.3, May 2012

conditions. The base station combines the relay station signal with the mobile station signal. Initially we assume no fading. We use BPSK and QPSK modulation schemes and study the effect of the modulation scheme being used. The model can be easily extended to QAM modulation scheme. We compare the BER results for different SNRs using QPSK and BPSK modulation schemes. Also we compare the BER results for without relay and with DF relay and MRC combining.

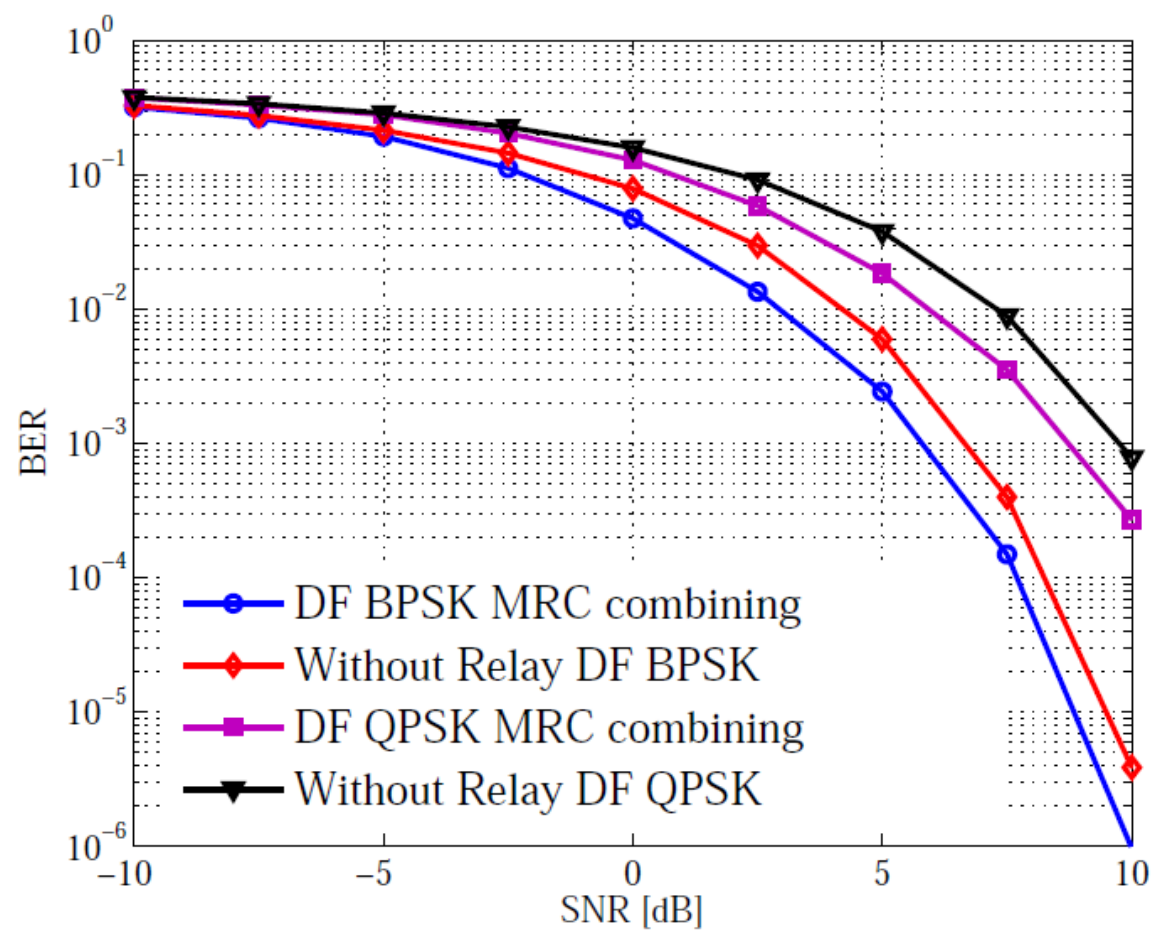

Figure 2. BER vs. SNR with DF relay and without relay with no fading

From the simulations on DF relay without fading as shown in Figure 2, we demonstrated that the BER performance of DF relay with MRC combining is better than that of without relay and without MRC combining. The BPSK modulation scheme has better performance over QPSK, which justifies the selection of BPSK as a modulation scheme.

The BER performance with Rayleigh fading is as shown in Figure 3. The BER performance degrades due to Rayleigh fading. The BER plots demonstrates the improved BER performance with DF relay and MRC combining. 


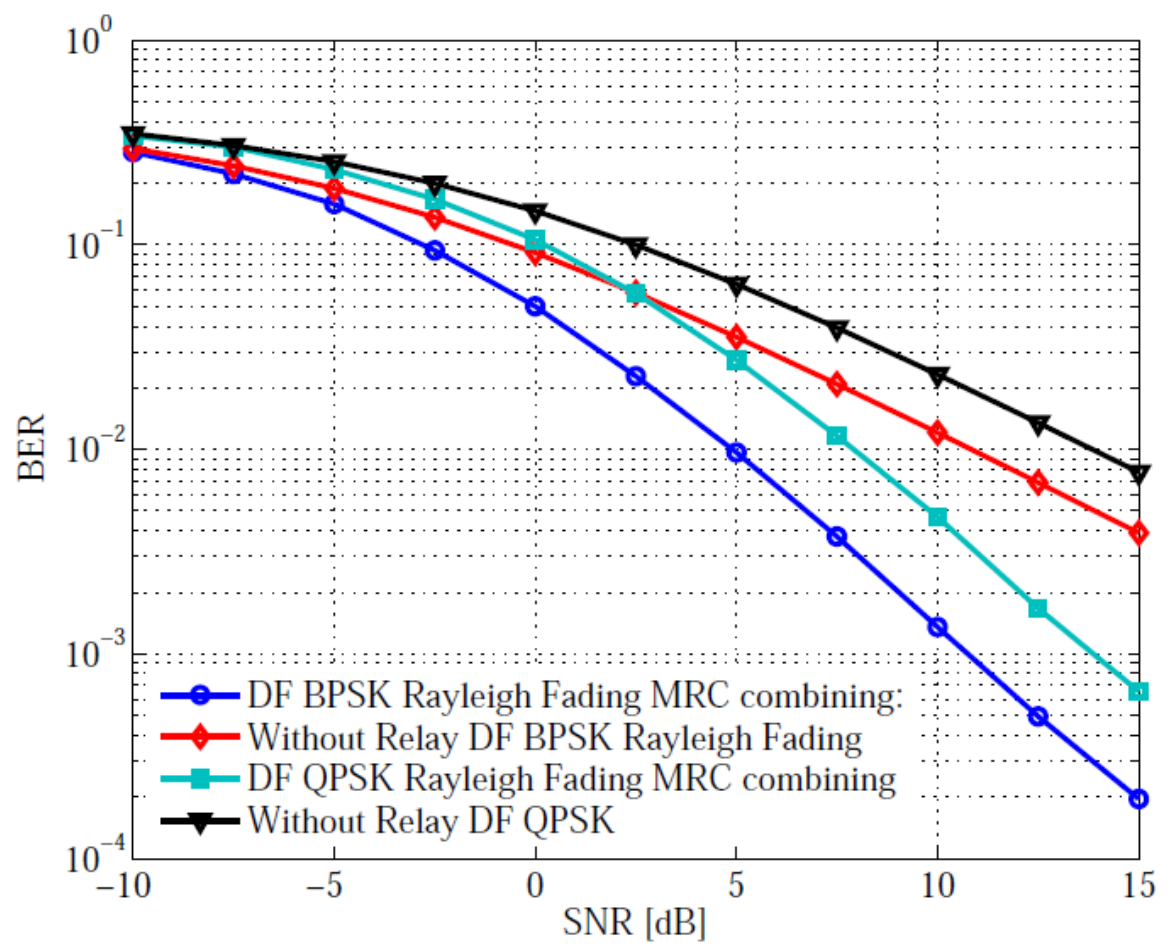

Figure 3. BER vs. SNR with DF relay and without relay with Rayleigh fading

\subsection{Effect of Packet Length and Packet Combining ARQ on Throughput Performance of Relay Network}

We apply packet combining ARQ to the proposed relay network scheme and investigate the throughput performance for different RS-BS channel conditions, which are indicated by different RS-BS SNRs.

Here, we discuss the simulation results to compare the throughput performance of the proposed scheme for different relay station to base station link conditions. The throughput is defined as the number of information bits carried per unit time. Here, we consider the useful information bits only to compute the throughput. The average throughput for MS-BS average SNR of 10 to $20 \mathrm{~dB}$ is observed for different RS-BS average SNR. 


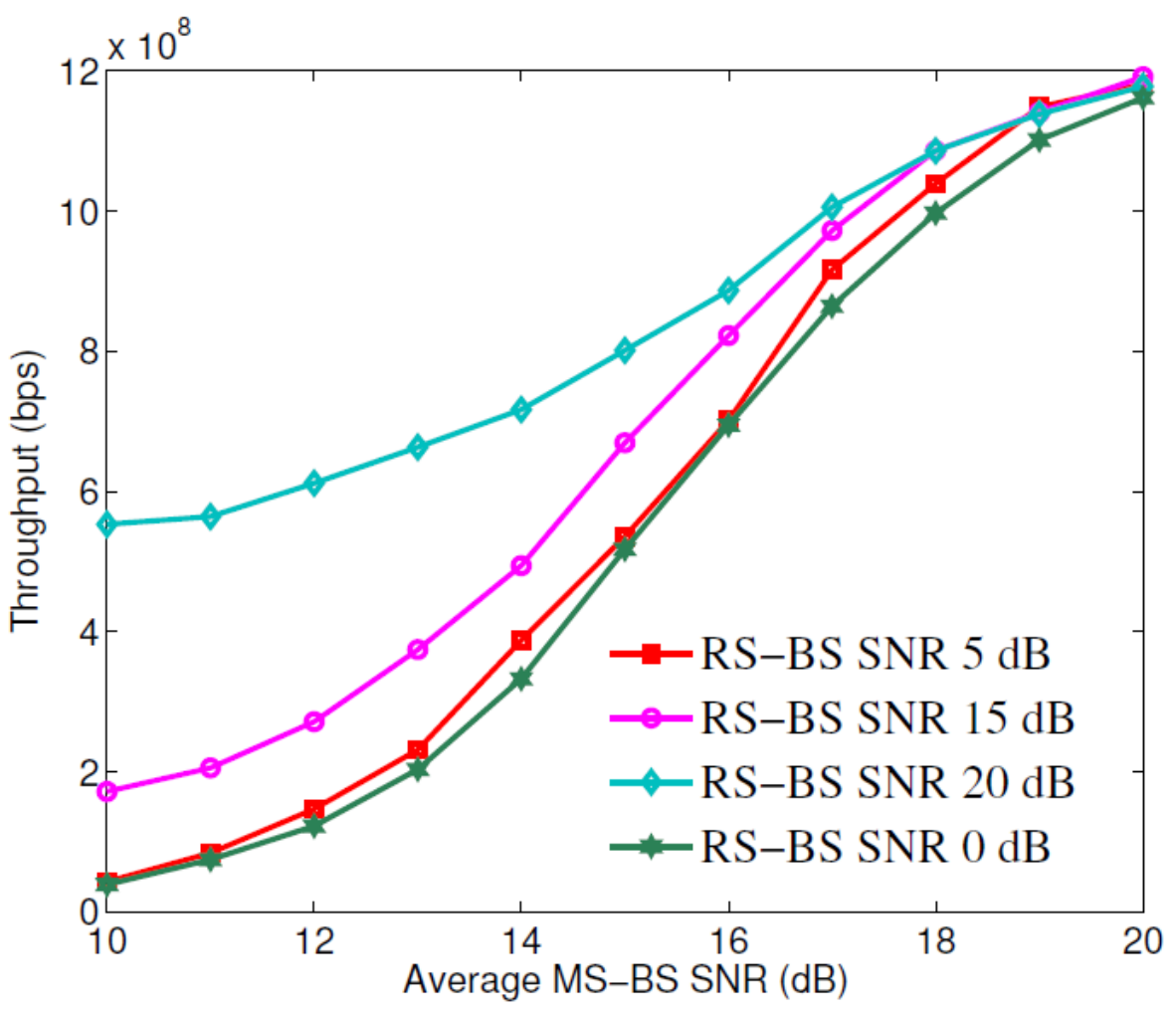

Figure 4. Average Throughput for different RS-BS channel conditions with different MS-BS SNR and payload $=20$ Bytes

We can see from Figure 4, that the throughput of the cooperative relay system is lower when the RS-BS SNR is lower. But when the RS-BS link has better SNR, the throughput of the system is improved. We can also observe that the improvement in throughput at lower MS-BS SNR is higher than that of at higher MS-BS SNR. The optimum relay placement strategy can improve the SNR on the RS-BS link. We can leverage the better RS-BS channel condition to enhance the system throughput. The incremental relaying saves the resources when the channel condition is good. The packet combining prevents the unnecessary retransmissions by recovering the correct packets by combining the erroneous packets, which results in increase in throughput.

The throughput plot for a payload size of 20 Bytes is shown in Figure. 4 . We can see the effect of change in payload size on throughput by comparing the plots shown in Figure 4 and Figure 5. Figure 5 shows the throughput for payload size of 60 Bytes. We can see improvement in throughput with increase in payload size. There is an optimum packet size which gives maximum throughput. The increase in packet size beyond the optimum size will give decreased throughput. We also demonstrated that the improvement in throughput at the lower side of SNR is significant than that of at the higher side of SNR. 


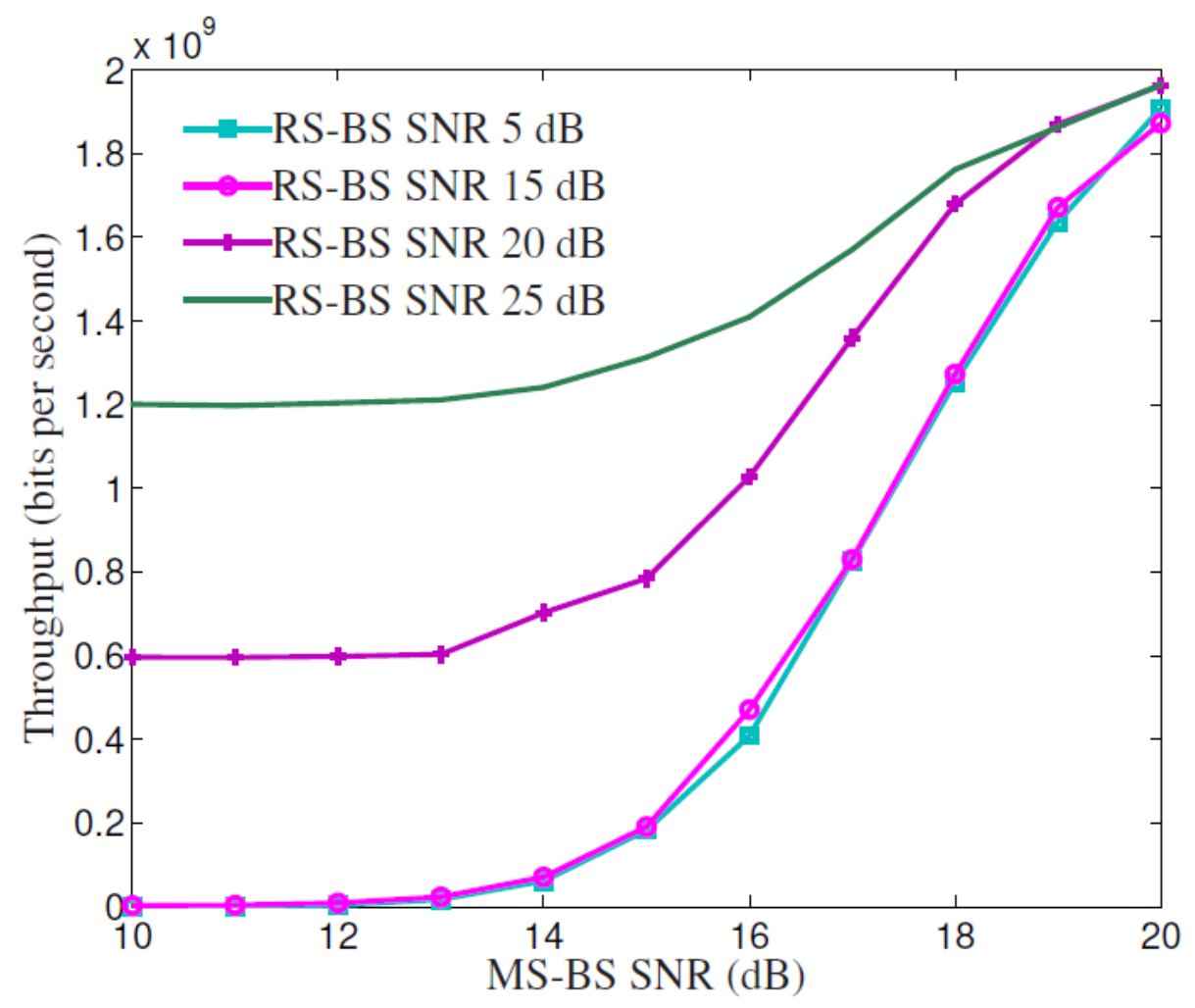

Figure 5. Average Throughput for payload of 60 Bytes

\section{Conclusions}

In this paper, the effect of packet combining and packet size is investigated in DF relay scenario. In our proposed scheme, the DF relay retransmits only if the direct transmission fails. This saves the resources by avoiding unnecessary transmissions and hence improving the throughput of the network in cooperative relay scenario. The algorithm developed here facilitates for packet combining in cooperative DF relay. We found that for fixed DF relay the BER performance with MRC combining is better than that of without MRC combining and relay. The performance in Rayleigh fading is investigated, where we found improvement with MRC and relay. The optimum relay placement strategy can improve the SNR on the RS-BS link. We can leverage the better RS-BS channel condition to improve the system throughput. We demonstrated that with better RS-BS link SNR the throughput in this scheme is improved. The packet combining reduces the number of retransmissions, improving the throughput. The change in packet length causes change in throughput. There is an optimum packet length which maximizes the system throughput.

\section{ACKNOWLEDGEMENTS}

This work is supported by the India-UK Advanced Technology Center of Excellence in Next Generation Networks (IU-ATC) project. 
International Journal of Computer Networks \& Communications (IJCNC) Vol.4, No.3, May 2012

\section{REFERENCES}

[1] J. Laneman, D. Tse, and G. Wornell, "Cooperative Diversity in Wireless Networks: Efficient Protocols and Outage Behaviour," IEEE Transcations on Information Theory, vol. 50, pp. 3062-3080, Dec 2004.

[2] R. Patil, P. Chaporkar, and A. Karandikar, "Diversity Combining and Packet Size Adaptation for Maximizing Throughput of ARQ Protocols in AWGN and Fading Channel," in IEEE International Conference on Ultra Modern Telecommunications, ICUMT 2009, October 2009.

[3] S. Ikki and M. H. Ahmed, "Performance Analysis of Incremental Relaying Cooperative Diversity Networks over Rayleigh Fading Channels," in IEEE Wireless Communications and Networking Conference, 2008. WCNC 2008., pp. 1311-1315, March 2008.

[4] T. Issariyakul and V. Krishnamurthy, "Amplify-and-Forward Cooperative Diversity Wireless Networks: Model, Analysis, and Monotonicity Properties,” IEEE/ACM Transcations on Networking, vol. 17, February 2009.

[5] H. Chen and M. H. Ahmed, "Throughput Enhancement in Cooperative Diversity Wireless Networks using Adaptive Modulation," in Canadian Conference on Electrical and Computer Engineering, 2008. CCECE 2008.

[6] M. Mardani, J. Harsini, and F. Lahouti, "Cross-layer link adaptation design for relay channels with cooperative arq protocol," in IEEE 10th Workshop on Signal Processing Advances in Wireless Communications, 2009. SPAWC '09, July 2009.

[7] Y. Luo and L. Cai, "Throughput Maximization for User Cooperative Wireless Systems with Adaptive Modulation,” in IEEE Conference on Communication, 2010. IEEE ICC, 2010.

[8] G. R. Woo, P. Kheradpour, and D. Katabi, "Beyond the Bits: Cooperative Packet Recovery Using Physical Layer Information,” in ACM MOBICOM, September 2007.

[9] N. Ahmed and B. Aazhang, "Throughput Gains Using Rate and Power Control in Cooperative Relay Networks," IEEE Transcations on Communications, vol. 55, pp. 656-660, April 2007.

[10] J. X. Chen and W. B. Wang, "ARQ strategies for relay cooperative systems," The Journal of China Universities of Posts and Telecommunications, vol. 14, October 2007.

[11] K. J. R. Liu, A. K. Sadek, W. Su, and A. Kwasinski, Cooperative Communications and Networking. Cambridge University Press, 2009.

[12] D. Tse and P. Vishwanath, Fundamentals of Wireless Communication. Cambridge University Press, 2005.

\section{Authors}

Rajendrakumar A. Patil is a Ph.D. Research Scholar in the Department of Electrical Engineering, Indian Institute of Technology Bombay, Mumbai, India. He holds a M.Tech. in Electronics and Communications Engineering from National Institute of Technology (NIT), Kurukshetra, India. He is a faculty at College of Engineering Pune, India. He has research and teaching experience of 23 years. His areas of interest are Wireless Communication, Communication Networks and protocols.

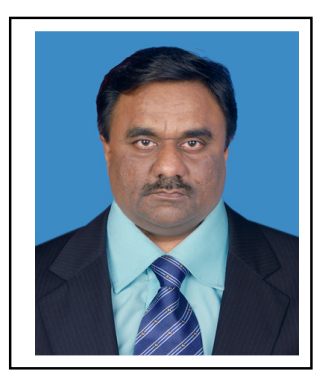

